\title{
MECHANISM OF HOUSE FLY RESISTANCE: II- BINARY MIXTURES OF CYPERMETHRIN, IMIDACLOPRID AND SPINOSAD AND THEIR IMPACT TO BREAK DOWN THE LABORATORY DEVELOPED RESISTANCE. Doaa El-Sherif ${ }^{1}$, Makram Sayed ${ }^{1}, Z_{\text {Zaki El-Fiky }}{ }^{2}$ and Ahmed Etman ${ }^{1}$ \\ 1. Plant Prtectin Dept, Faculty of Agric. Fayoum Univ. \\ 2. Genetic Dept. Faculty of Agric., Faayoum Univ.
}

\begin{abstract}
The present study indicated that the repeated exposure of the laboratory strain of the house fly, Musca domestica (Linnaeus) to Cypermethrin, Imidacloprid and Spinosad lead to developing resistance against these insecticides. The resistance level of the selected strains reached after $30^{\text {th }}$ generation to $24.8,15.53$ and 9.56 folds for Cypermethrin, Imidacloprid and Spinosad compared with the laboratory strain.

This study also showed the breakdown of resistance using insecticide binary mixtures, where the mixture of Cypermethrin+ Imidacloprid exhibited potentiation at the ratio of 1:1 and 1:2 against all the resistant strains. Also, the combination of Cypermethrin with Spinosad gave potentiation at the ratios of $1: 1,1: 2$ and 2:1 against Imidacloprid and Spinosad resistant strains.
\end{abstract}

Key words: Resistance, Joint action, cypermethrin, imidacloprid, spinosad

\section{INTRODUCTION}

Management of the house fly requires multiple applications of different insecticides. Unfortunately, the house fly has a well-documented history of developing resistance to many insecticides, including pyrethroids, neonicotinoids, organophosphates (OPs), carbamates, organochlorines and the triazine cyromazine. The house fly has been found to be resistant to 62 unique insecticide active ingredients, with 337 documented cases worldwide, and is listed as the world's No. 1 resistant urban insect pest (Zhu, et al., 2016 and Li, et al., 2013). Increased metabolic detoxification and decreased target site sensitivity of the insect nervous system are two of the major mechanisms involved in the development of insecticide resistance in house flies ( $\mathrm{Li}$, et al., 2011).

The current investigation is curried out using the insecticides formulation Cymbush $^{\circledR}$ 10\% EC (Cypermthrin), Imidazed ${ }^{\circledR} 20 \%$ SC) (Imidacloprid) and Tracer $^{\circledR} 24 \%$ SC (Spinosad) insecticides on $M$. domestica . The adult house fly resistance to Cypermethrin, Imidacloprid and Spinosad was developed in the laboratory through 30 generation. The aim of this study is to using the binary mixtures of the tested insecticides to breakdown the laboratory developed resistance.

Fayoum J. Agric. Res. \& Dev., Vol. 33, No.2, July, 2019 
Doaa El-Sherif ${ }^{1}$, et al.,

MATERIAL AND METHODS

\section{Development of resistant strains}

The laboratory strain of $M$. domestica was reared in the insects rearing room, Plant Protection Department, Faculty of Agriculture, Fayoum University using different media according to (Singh and Jerram, 1976 and ElSherif, et al., in press). The laboratory strain was used to build up the resistance against the insecticides, Cypermethrin, Imidacloprid and Spinosad. Adults were treated with the calculated $\mathrm{LC}_{50}$ values of Cypermthrin, Imidacloprid and Spinosad (El-Sherif, et al., in press). Survivors after $24 \mathrm{~h}$ treatment were used to establish a new generation for each tested insecticide. Each generation was exposed to the same $\mathrm{LC}_{50}$ until mortality reached a rate less than $35 \%$, at this point a $\mathrm{LC}_{50}$ was estimated from a new dose-response curve. This procedure was followed for 30 generation, and the resistance ratio (Fold) was calculated using the following formula (Georghiou 1962) until the thirty generation.

$$
\text { Fold }=\frac{\text { LC50 of resistant strain }}{\text { LC50 of laboratory strain }}
$$

\section{Effect of binary mixtures of different insecticides}

The adult's medium (black honey and dry yeast powders at the ratio of 1:2) were applied to evaluate the efficiency of the binary mixtures of different insecticides to investigate their role in breakdown of the house fly resistance against insecticides.

The calculated values of $\mathrm{LC}_{25}$ of the Cypermethrin, Imidaclprid and Spinosad were used to make the binary mixtures at the rate of 1:1, 1:2 and 2:1. The prepared mixture were added to the adult's media to get poison bait, which introduced to the house fly adults. Percent mortality was recorded at $24 \mathrm{~h}$ post treatment and the co-toxicity factor was estimated according to Mansour, et al., ( 1966 and 2010) equation.

The co-toxicity factor with a positive value from 20 or more indicates potentiation, a negative factor of -20 indicates antagonism, and the intermediate values of $>-20$ to $<20$ indicates an additive effect.

\section{RESULTS AND DISCUSSION}

\section{Resistance to Cypermethrin}

Data in Table (1) and Fig. (1) show the development of resistance to Cypermethrin expressed as $\mathrm{LC}_{50}$ values and resistance ratio (fold). The selection process by Cypermerthin in the laboratory was carried out until the thirtieth generation. The house fly resistance increased against Cypermethrin and recorded as 24.8 fold in the generation 30 compared with the laboratory strain.

Development of Cypermethrin resistance in the current study was slowly compared with the other studies. High levels of resistance to the pyrethriod detected by Abbas and Shad, (2015), where they reported that,

Fayoum J. Agric. Res. \& Dev., Vol. 33, No.2, July, 2019 
MECHANISM OF HOUSE FLY RESISTANCE: II- BINARY.

house fly developed 445 fold resistances against Lambda-Cyhalothrin after 26 generations. Also, Deltamethrin selected strain for six generations built a resistance ratio (RR) of 176.34-fold, when compared with the lab-susceptible strain, (Khan, et al., 2015).

Table (1). The values of $\mathrm{LC}_{25}, \mathrm{LC}_{50}(\mathrm{ppm})$, slope and fold values of resistance in laboratory and the three resistant strains at $30^{\text {th }}$ generation

\begin{tabular}{|c|c|c|c|c|c|}
\hline Insecticides & Strains & $\mathbf{L C}_{\mathbf{2 5}}$ & $\mathbf{L C}_{\mathbf{5 0}}$ & Slope & RR* (Fold) $^{-}$ \\
\hline \multirow{2}{*}{ Cypermethrin } & Laboratory & 89.5 & 208.75 & 1.94 & - \\
\cline { 2 - 6 } & Resistant & 2200 & 5177.09 & 2.33 & 24.8 \\
\hline \multirow{2}{*}{ Imidacloprid } & Laboratory & 125 & 238.3 & 2.41 & - \\
\cline { 2 - 6 } & Resistant & 1740 & 3700.59 & 1.95 & 15.53 \\
\hline \multirow{2}{*}{ Spinosad } & Laboratory & 41 & 86.05 & 2.34 & - \\
\cline { 2 - 6 } & Resistant & 410 & 822.78 & 2.13 & 9.56 \\
\hline
\end{tabular}

*Resistance ratio $(\mathbf{R R})=\mathrm{LC}_{50}$ of resistant strain $/ \mathrm{LC}_{50}$ of laboratory strain

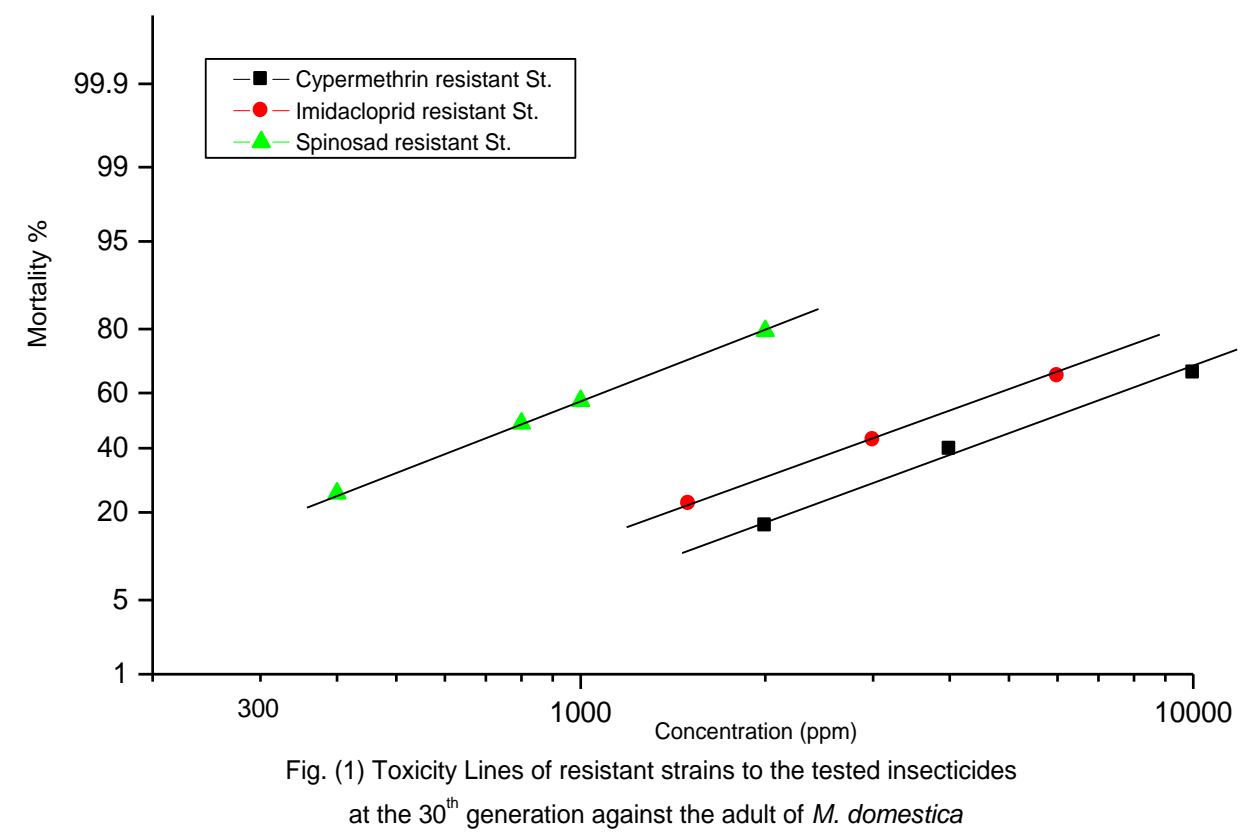

\section{Resistance Imidacloprid in M. domestica}

Data in Table (1) and Fig. (1) show that the Imidacloprid selected population developed 15.53 fold resistance in the thirtieth generation compared with the laboratory population. This means that $M$. domestica showed moderate resistance to Imidacloprid. In contrast, Khan, et al., (2014)

Fayoum J. Agric. Res. \& Dev., Vol. 33, No.2, July, 2019 
Doaa El-Sherif ${ }^{1}$, et al.,

found that after 13 generations of laboratory selection with Imidacloprid, the resistance ratio increased to 106 fold compared with the susceptible strain and Kaufman, et al., (2010) found that high level of resistance after the fifth generation selection, was 331-fold at the $\mathrm{LC}_{70}$ were recorded.

Ma, et al., (2017) reported that, Imidacloprid-resistant strain was established from the field strain by their selections with Imidacloprid for 21 generations in laboratory, showing 80.15-fold compared with the susceptible strain.

\section{Resistance Spinosad in M. domestica}

Low level resistance was observed for Spinosad in $M$. domestica, where the resistance ratio did not reach 10 fold after thirtieth generation under Spinosad selection (Table 1 and Fig. 1). This finding could be due to the absence and or inactive metabolic and genetic factors, which develop the Spinosad resistance. Also, this fact could promote the using of Spinosad in the control process against the house fly. Building up of resistant to Spinosad increased slowly, where the resistance ratio recorded 9.56 fold at thirtieth generation. Shi, et al., (2011) reported that the house fly exhibited 279 fold after 27 generation compared with the susceptible strain. In addition, Shono and Scott, (2003), found that the selection of the houseflies produced 150 fold against Spinosad after 10 generations of selection.

\section{Effect of binary mixtures of the tested insecticides against resistant strains after 24h of exposure.}

The interaction effects among the tested insecticides depended on the type of insecticides used, ratios and strains. The values $\mathrm{LC}_{25}$ of the Cypermethrin, Imidacloprid and Spinosad were used to make the binary insecticide combinations, where calculated in the resistant strains after the $30^{\text {th }}$ generation, 2200, 1740 and 410 ppm, respectively.

Data in (Table 2 and Fig. 2) show the mixture insecticides in different strains; the mixture of Cypermethrin+Imidacloprid showed potentiation when used at the ratio of 1:1 and 1:2 in all the strains and it gave high potentiation. While at a ratio of 2:1 it showed an additive effect in the Cypermethrin and Imidaclporid resistant strains and potentiation in Spinosad resistant strain. This could be because the result of different modes of action they poses, or because the binding of monooxygenase enzymes with Imidacloprid insecticide would prevent or delay the degradation, and enhance the toxicity of Cypermethrin by competitive substrate inhibition mechanism. As is the case in some organophosphate insecticides which bind to the active site associated with esterase enzymes responsible for detoxification of pyrethroid-based insecticides (Cloyd, 2011 and Ahmad, 2009).

The mixture of Cypermethrin with Spinosad gave the highest cotoxicity factors Imidacloprid and Spinosad resistant strains at the ratio of 1:1, recording 54 and 36, respectively. In addition to, it gave potentiation in the other ratios in Imidacloprid and Spinosad resistant strains, and also, showed

Fayoum J. Agric. Res. \& Dev., Vol. 33, No.2, July, 2019 
potentiation at a ratio of 1:2 against the Cypermethrin resistant strain, but it showed an additive effect when used at the ratio of 1:1 and 2:1 in the same strain. This is useful in preventing the development of resistance in $M$. domestica to the tested insecticides. The toxicity of pyrethroids could be enhanced by the addition of new insecticides like Emamectin benzoate and Fipronil and Spinosad. According to this, one toxicant in the mixture interferes with the metabolic detoxification of the other toxicant, Khan, et al., (2013) they demonstrated that the mixture of Cypermethrin+Emamectin gave a synergistic action. These results conversely agree with Abbas, et al., (2015) whom revealed that combination indices for Lambda-Cyhalothrin+Emamectin benzoate and Lambda-Cyhalothrin+ Spinosad mixtures were significantly less than 1, demonstrating an antagonistic effect. Thus, Vayias, et al., (2010), revealed that the combination of Spinosad with Deltamethrin did not appear to be compatible with the rice weevil, Sitophilus oryzae (Linnaeus).

The potentiation effect was also demonstrated in the case of the combination between pyrethroids and organophosphates by (Zahidul and Khalequzzaman, 2002 and Asid, et al., 2017).

On the other hand, the mixture of Imidaclporid+Spinosad showed an additive effect at all mixing ratios and also all the tested strains expect in the Imidacloprid resistant strain at the ratio of $1: 1$ which gave a potentiation effect. Also, the mixture containing Fipronil and Acetamiprid have an additive effect on M. domestica (Levchenko, et al., 2018).

Table (2): Efficacy of binary mixtures against Cypermethrin, Imidacloprid and Spinosad resistant strains of M. domestica.

\begin{tabular}{|c|c|c|c|c|}
\hline \multirow{2}{*}{ Combinations } & \multirow{2}{*}{ Mixing ratio } & \multicolumn{3}{|c|}{ Co-toxicity factor } \\
\hline & & Cypermethrin & Imidacloprid. & Spinosad \\
\hline \multirow{3}{*}{$\begin{array}{l}\text { Cypermethrin+Imid } \\
\text { acloprid }\end{array}$} & $1: 1$ & $36 \mathrm{P} *$ & $38 \mathrm{P}$ & $32 \mathrm{P}$ \\
\hline & $1: 2$ & $29.3 \mathrm{P}$ & $29 p$ & $22.7 \mathrm{p}$ \\
\hline & $2: 1$ & $6.7 \mathrm{Ad}^{* *}$ & $16 \mathrm{Ad}$ & $21.3 \mathrm{P}$ \\
\hline \multirow{3}{*}{$\begin{array}{l}\text { Cypermethrin+Spin } \\
\text { osad }\end{array}$} & $1: 1$ & $8 \mathrm{Ad}$ & $54 \mathrm{P}$ & $36 \mathrm{P}$ \\
\hline & $1: 2$ & $22.7 \mathrm{P}$ & $29.3 \mathrm{P}$ & $25.3 \mathrm{P}$ \\
\hline & $2: 1$ & $4 \mathrm{Ad}$ & $22.7 \mathrm{P}$ & $25.3 \mathrm{P}$ \\
\hline \multirow{3}{*}{$\begin{array}{l}\text { Imidacloprid+ } \\
\text { Spinosad }\end{array}$} & $1: 1$ & $20 \mathrm{Ad}$ & $22 \mathrm{P}$ & $8 \mathrm{Ad}$ \\
\hline & $1: 2$ & $5.3 \mathrm{Ad}$ & $18.7 \mathrm{Ad}$ & $20 \mathrm{Ad}$ \\
\hline & $2: 1$ & $4 \mathrm{Ad}$ & $-6.7 \mathrm{Ad}$ & $1.3 \mathrm{Ad}$ \\
\hline
\end{tabular}

*P. Potentiation effect

**Ad. Additive effect

Fayoum J. Agric. Res. \& Dev., Vol. 33, No.2, July, 2019 


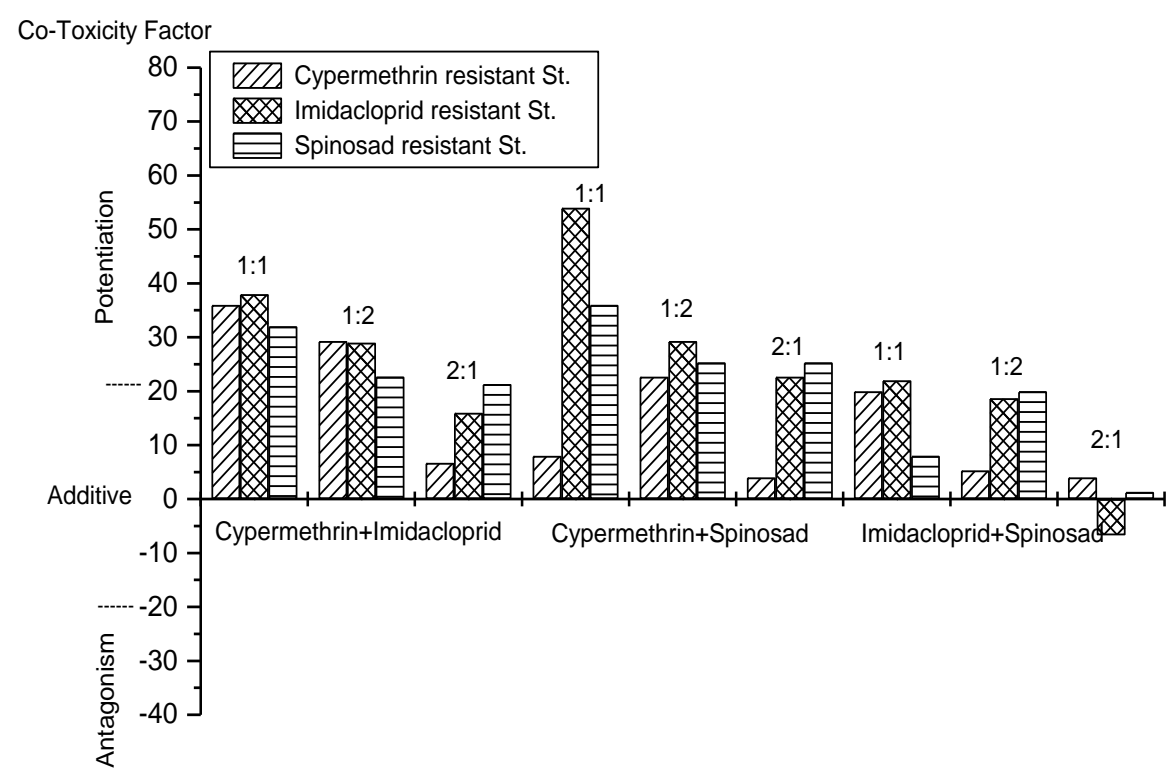

Fig. (2) The joint action of the tested insecticides against the Cypermethrin, Imidacloprid and Spinosad resistant strains of house fly.

\section{REFERENCES}

Abbas, N. and S. A. Shad (2015). Assessment of resistance risk to Lambdacyhalothrin and cross-resistance to four other insecticides in the house fly, Musca domestica L. (Diptera: Muscidae). Parasitol Res., 114: 2629-2637.

Abbas, N.; N. Crickmore and S. A. Shad (2015). Efficacy of insecticide mixtures against a resistant strain of house fly (Diptera: Muscidae) collected from a poultry farm. J. Trop insect sci., 35: 48-53.

Ahmad, M. (2009). Observed potentiation between pyrethroid and organophosphate insecticides for the management of Spodoptera litura (Lepidoptera: Noctuidae). Crop Protect., 28: 264-268.

Asid, A. N.; K.M. Al-Ghamdi.; A. A. H Mangoud.; K. Al Asiry.; N. A Alkenani and Y. Anwar (2017). Synergistic effect of insecticides on the larvae and adults of housefly, Musca domestica L. J. Entomol Zool., 5: 899-905.

Cloyd, R. A (2011). Pesticide mixtures, pesticides - Formulations, Effects, book. ISBN: 978-953-307-532-7.

El-Sherif, D.; M. Sayed.; Z. El-Fiky and A. Etman (In press). Toxicity of Cypermethrin, Imidacoloprid and Spinosad to larvae and adults of the laboratory strain. Fayoum J. Agric. Res. \& Dev.

Fayoum J. Agric. Res. \& Dev., Vol. 33, No.2, July, 2019 
Georghiou G. P (1962). Carbamate insecticides: toxic action of synergized carbamates against twelve resistant strain of the house fly. $J$. Economic Entomol., 55: 768-772.

Kaufman, P. E.; S. C. Nunez.; C. J. Geden and M. E. Scharf. (2010). Selection for Resistance to Imidacloprid in the House Fly (Diptera: Muscidae). J. Econ. Entomol., 103: 1937-1942.

Khan, H.A.A.; W. Akram.; S.A Shad and J. Lee (2013). Insecticide mixtures could enhance the toxicity of insecticides in a resistant dairy population of Musca domestica L. Plos one, 13(4): 1-8.

Khan, H.; N. Abbas.; S. A. Shad and M. B. S. Afzal (2014). Genetics and realized heritability of resistance to Imidacloprid in a poultry population of house fly, Musca domestica L. (Diptera: Muscidae) from Pakistan. Pestic. Biochem. Physiol., 114: 38-43.

Khan, H. A.; W. Akram and M. S. Haider (2015). Genetics and mechanism of resistance to deltamethrin in the house fly, Musca domestica L., from Pakistan. Ecotoxicol., 24: 1213-1220.

Levchenko, M.A.; E. A. Silivanova.; R.K. Bikinyaeva and G. F. Balabanova (2018). Efficacy of Acetamiprid and Fipronil fly baits against the housefly (Musca domestica L.) under laboratory conditions. Veterinary World, 11: 953-958.

Li T.; C. Cao; L. He; M. Li; L. Zhang; L. Zhang; H. Liu and N. Liu (2011). Autosomal interactions and mechanisms of pyrethroid resistance in house flies, M. domestica. Inter. J. Biol. Sci., 7: 902911.

Li, M.; W. R Reid; L. Zhang; J. G Scott; X. Gao; M. Kristensen and N. Liu (2013). A whole transcriptomal linkage analysis of gene coregulation in insecticide resistant house flies, Musca domestica. BMC Genomics, 14: 1471-2164.

Ma, Z.; J. Li.; Y. Zhang.; C. Shan and X. Gao (2017). Inheritance mode and mechanisms of resistance to Imidacloprid in the house fly Musca domestica (Diptera:Muscidae) from China. PLos one, 12: 1-15.

Mansour, N. A.; M. E. El-Defrawi.; A. Toppozada and M. Zied (1966). Toxicological studies on the Egyptian cotton leaf worm Prodenia litura, potentiation and antagonism of organophosphorus and carbamate insecticides. J. Econ. Entomol., 59(2): 307-311.

Mansour, S. A.; R. F.A. Bakr.; L. S. A. Hamouda and R. I. Mohamed (2010). Toxic and synergistic properties of several botanical extracts against larval and adult stages of the mosquito, Anopheles. pharoensis Bio. pestic. Int, 6: 129-145.

Shi, J.; L. Zhang and X. Gao (2011). Characterisation of Spinosad resistance in the housefly Musca domestica (Diptera:Muscidae). Pest Manag Sci., 67: 335-340.

Fayoum J. Agric. Res. \& Dev., Vol. 33, No.2, July, 2019 
Doaa El-Sherif ${ }^{1}$, et al.,

Singh, P. and E.M. Jerram (1976). Rearing house fly larvae in polythen bags. J. Zool., 3: 37 -58.

Shono, T. and J. G. Scott (2003). Spinosad resistance in the house fly, Musca domestica, is due to a recessive factor on autosome 1. Pestic. Biochem. Physiol., 75: 1-7.

Vayias, B.J.; N.G. Kavallieratos.; C.G. Athanassiou and G. Tatsi (2010). Insecticidal action of the combined use of Spinosad and Deltamethrin against three stored product pests in two stored hardwheat varieties. Crop Protect., 425: 919-922.

Zahidul, I. M and M. Khalequzzaman (2002). Potentiation of Malathion by other insecticides against adult house fly. Pakistan J. Biol. Sci., 5: 299- 302.

Zhu, F.; L. Lavine; S. O’Neal; M. Lavine; C. Foss and D. Walsh (2016). Insecticide resistance and management strategies in urban ecosystems. Insecta, 7: 1-26.

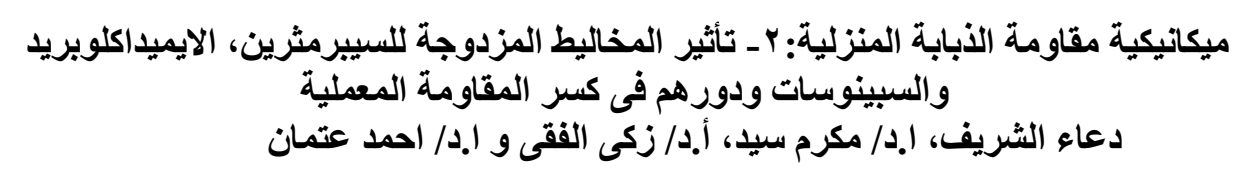

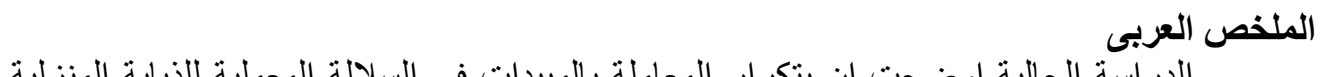

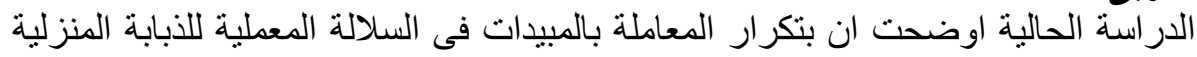

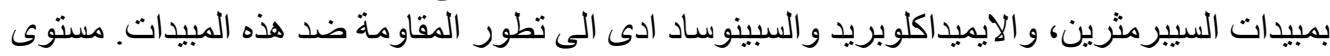

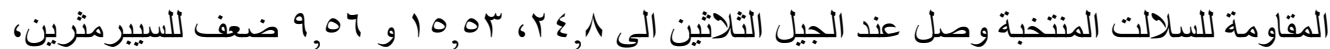

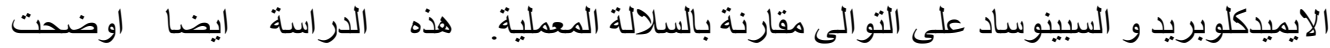

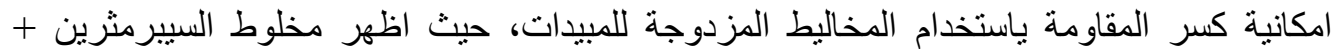

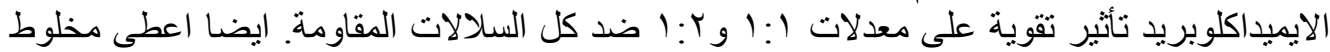

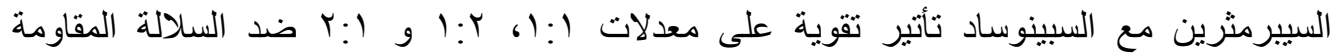

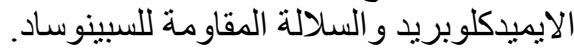

Fayoum J. Agric. Res. \& Dev., Vol. 33, No.2, July, 2019 